\title{
Système de santé et de soins infirmiers en France
}

\author{
Health and nursing system in France
}

Christine Guesdon-Caltero (http://orcid.org/0000-0002-5589-5716) ${ }^{1}$

Nadia Cherchem (http://orcid.org/0000-0003-0256-0848) ${ }^{1}$

Mirna Albuquerque Frota (http://orcid.org/0000-0003-3004-2554) ${ }^{2}$

Karla Maria Carneiro Rolim (http://orcid.org/0000-0002-7914-6939) ${ }^{2}$

${ }^{1}$ Espace Régional de

Formation des Professions de Santé. Centre Hospitalier

Rouen Normandie

Université. Rue de

Professeur Stewart 14. 76042

Rouen França. christine.

caltero@chu-rouen.fr

${ }^{2}$ Universidade de Fortaleza.

Fortaleza CE Brasil.

\begin{abstract}
This paper concerns the French Health Care System that revolutionized the rationale of the existing social protection. It emphasizes that, in this country, it is essential to train professionals integrated into a "know-how", and also prepared for the current and future changes in the nursing profession. A literature review was carried out based on the classification and analysis of the texts and documents researched, and we tried to understand the French Health and Nursing System. This allowed the construction of two thematic categories. The first one is the "Health Service" that addresses health training courses for prevention, while the second one deals with Nursing Education, which is a competency-based framework. The demography of nurses in France is also discussed, which highlights the increased number of nurses from 2010 to 2018.
\end{abstract}

Key words Nursing, Professional training, Labor market
Résumé Cet article concerne le Système de Santé en France. Il souligne que dans ce pays il est essentiel de former des professionnels insérés dans un «savoir-faire», et également préparés aux mutations actuelles et à venir de la profession infirmière. Ainsi, une revue de la littérature a été réalisée à partir de la classification et de l'analyse des textes et des documents recherchés, nous avons essayé de comprendre le Système de Santé Français et de soins infirmiers. Cela a permis la construction de deux catégories thématiques. La première est le Service Sanitaire réalisé par les étudiants des formations en santé qui traduit la volonté du Gouvernement de placer la prévention au cœur de son action. Et la deuxième aborde la Formation des infirmiers qui est un référentiel basé sur la compétence. La démographie des infirmières en France est également abordée, ce qui met en évidence l'augmentation du nombre d'infirmières de 2010 à 2018.

Mots-Clés Soins Infirmiers, Formation professionnelle, Marché du travail 


\section{Introduction}

La France, à l'instar des autres pays européens, dispose d'un système de santé universel, largement financé par l'État à travers un système national d'assurance maladie. Dans l'évaluation mondiale des systèmes de santé réalisée en 2000, l'Organisation mondiale de la santé avait conclu que la France fournissait «les meilleurs soins de santé globaux» du monde ${ }^{1}$.

Le système de santé publique français intègre des régimes de sécurité sociale complets, mis en place pour garantir l'accès aux droits considérés comme fondamentaux par l'ensemble de la population, tels que la santé, le bien-être social, les soins de santé et le droit du travail. Basés sur des principes d'équité en matière de couverture et de solidarité en matière de financement, ce système a révolutionné la logique de la protection sociale existante. Dans le secteur de la santé, il s'appuyait sur des programmes de philanthropie et d'assurance individuelle contributifs limités à une partie seulement de la population (en particulier les travailleurs et leurs personnes à charge). Malgré ces similitudes, ces systèmes ont des voies de consolidation et d'articulation différentes avec le secteur privé2.

La révélation de documents sur l'histoire des sciences infirmières dans différentes régions du monde s'est révélée être une pratique de la connaissance de soin, étant donné que la relation de la profession infirmière avec la société est pleine de concepts, qui ont été établis dans la trajectoire historique de la profession et influencent la conception de sa signification en tant que profession de la santé.

La profession d'infirmière brésilienne ne diffère pas beaucoup de la réalité française. Padilha et Borenstein décrivent que les soins infirmiers en France ont été marqués par une histoire de lutte pour la reconnaissance, l'appréciation et le développement de la profession, aboutissant à des réalisations pouvant donner des orientations nouvelles et décisives à la profession. La connaissance des courants socio-économiques, culturels et politiques qui influencent et ont influencé le cours de l'histoire de la profession d'infirmière permet d'élucider le contexte de changements dans la pratique ${ }^{4}$.

Selon Courtois et al. ${ }^{5}$, la professionnalisation des soins infirmiers en France s'est produite parallèlement à l'instauration de la Troisième République (1870-1940), période qui a entraîné une laïcité progressive des institutions. Sœurs de la Charité qui travaillaient dans des hôpitaux.
Dans ce contexte, deux projets de formation d'infirmières ont vu le jour: le modèle républicain des écoles d'assistance publique de Paris et le modèle rossignol distribué par la province de France. La première souligne le besoin de l'infirmière de servir le patient et le modèle anglo-saxon, fondé sur une organisation collective et structurée, avec des outils tels que le dossier de soins, l'élaboration de diagnostics, le positionnement flexible entre l'infirmière et le patient, la poursuite de l'autonomie et de la compétence technique ${ }^{5}$.

En France, il est de plus en plus préoccupant de former des professionnels insérés dans un «savoir-faire», et préparés aux mutations que la profession a subies récemment et à venir. Ce fait est perçu par le moment historique de la profession infirmière et par la formation récente du Conseil national de l'Ordre des infirmières, organisme officiel qui représentait depuis janvier 2009 la profession d'infirmière dans ce pays ${ }^{6}$.

Ainsi, à partir de la classification et de l'analyse des textes et des documents recherchés, nous avons cherché à interpréter le système de santé et de soins infirmiers en France, ce qui nous a permis de construire trois catégories thématiques: Le Service Sanitaire: Les Formations en Santé au Service de la Prévention. - La Formation d'infirmiers en France: Un référentiel basé sur la compétence. - La Démographie des Infirmiers en France.

\section{Le Service Sanitaire: Les Formations en Santé au Service de la Prévention}

«Pour que le plus souvent possible, les Françaises et les Français tout au long de la vie puissent avoir à l'école, sur le lieu de travail, et encore plus que d'autres dans les zones défavorisées ou dans certains déserts médicaux, accès à la santé et aux soins, nous devons conduire une réforme profonde. Ce que je veux faire pour cela c'est créer un service sanitaire de trois mois pour les étudiants en santé (...). C'est un décloisonnement, c'est une révolution culturelle que nous devons conduire. Pour Emmanuel Macron, président de la République, ce service sanitaire sera intégré dans les maquettes pédagogiques, et, à terme, il pourra concerner plus de 40.000 étudiants chaque année» (Emmanuel Macron, discours à Nevers le 6 janvier 2017).

Engagement du Président de la République, le Service Sanitaire traduit la volonté du Gouvernement de placer la prévention au cœur de son action. Cela, avec un double objectif: initier à la prévention primaire ceux qui seront les profes- 
sionnels de santé de demain et leur permettre d'intervenir sur le terrain, auprès de la population, notamment les jeunes, pour réduire les inégalités sociales et territoriales de santé.

Dans une enquête en 2017, 12\% des jeunes de 17 ans indiquaient consommer de l'alcool plusieurs fois par jour et 25\% fumer quotidiennement. L'obésité pédiatrique touchait 3,5\% des enfants et était 4,5 fois plus fréquente chez les enfants d'ouvriers que les enfants de cadres. Enfin, $80 \%$ des adultes étaient sédentaires.

Ce constat inquiétant démontrait la fréquence élevée des comportements à risque en France et plus particulièrement chez les jeunes et les populations plus fragiles socialement.

Une politique d'informations et de prévention s'avérait donc être une nécessité.

Ainsi, le 26 mars 2018, le premier Ministre Edouard Philippe et la Ministre de la Santé Agnès Buzyn ont présenté les 25 mesures phares du Plan National de Prévention en matière de Santé. Parmi celles-ci la création du service sanitaire était annoncée. Le service sanitaire est un ensemble d'actions de prévention réalisées par les étudiants des filières de la Santé afin de promouvoir les comportements favorables à la Santé et sera déployé sur tout le territoire et auprès de tout type de public et plus particulièrement les plus fragiles.

Porté conjointement par le Ministère des Solidarités et de la Santé et le Ministère de l'Enseignement Supérieur, de la Recherche et de l'Innovation, le Service Sanitaire est mis en place pour la rentrée de septembre 2018 pour tous étudiants en Santé. Sont concernées dans un premier temps, les formations en médecine, odontologie, maïeutique, pharmacie, kinésithérapie et soins infirmiers, soit 47.000 étudiants par an. Pour la rentrée de septembre 2019, le Service Sanitaire sera étendu à l'ensemble des filières en Santé, ce qui portera à 50.000 étudiants par an.

La prévention passe notamment par la promotion de comportements favorables à la Santé dont l'impact sur la qualité de vie, sur la morbidité et sur la mortalité est démontré. Aujourd'hui, les différences d'espérance de vie, et plus généralement les inégalités sociales de Santé sont importantes et sont souvent liées à un accès inégal aux comportements favorables. La compétence à mener des actions de prévention et de promotion de la santé est donc primordiale et doit être impérativement partagée par l'ensemble des professionnels de santé.

Dans le cadre d'un module pédagogique de 6 semaines intégré dans la formation, le service sanitaire prendra la forme d'interventions de pré- vention, le plus souvent sous forme collective, sur des thèmes prioritaires de Santé Publique.

\section{La prévention comme enjeu...}

Conçu pour pallier le manque cruel de formation à la prévention durant les études, le Service Sanitaire a pour but d'initier les futurs professionnels de santé à l'animation d'ateliers préventifs en lien avec une thématique spécifique.

Le Service Sanitaire vise donc à former et familiariser les étudiants aux enjeux de la prévention primaire mais aussi à leur permettre de travailler ensemble autour d'un même thème dans le cadre de leur scolarité.

Grâce à des partenariats, les étudiants pourront ainsi découvrir en équipe, pluridisciplinaire ou non, d'autres facettes de leurs disciplines médicales au sein de structures où la santé n'est pas forcément une priorité telle que les collèges, les lycées mais aussi les entreprises et les milieux carcéraux. L'accent sera effectivement porté aux milieux et zones sensibles ou la tendance à un renoncement aux soins est la plus importante.

Pour ce faire, un premier temps de formation spécifique permet aux étudiants d'acquérir les connaissances et compétences nécessaires à l'intervention auprès des publics «cible».

En s'appuyant sur le rapport du Professeur Vaillant, mandaté par les Ministres des Solidarités et de la Santé ainsi que de l'Enseignement Supérieur, de la Recherche et de l'Innovation, les thèmes de prévention retenus sont: l'alimentation (la nutrition tout au long de la vie), l'activité physique, les addictions (alcool, tabac, cannabis et autres drogues illicites), la vie affective et sexuelle (éducation à la sexualité intégrant la prévention des infections et maladies sexuellement transmissibles ainsi que la contraception).

Ces thématiques ont été choisies pour leur impact sur la Santé en fonction des priorités nationales de Santé Publique et des politiques régionales afin notamment de mettre en adéquation l'offre de service sanitaire et les lieux d'intervention.

Les interventions des étudiants se déroulent dans: les Etablissements d'Enseignement primaire, secondaire et supérieur, les Centres de Formation Militaire, les Etablissements de santé et médico-sociaux (Etablissements d'Hébergement pour Personnes Agées et Dépendantes, maisons et centres de santé), les structures d'accompagnement social (crèches, centres de Protection Maternelle et Infantile, centres d'hébergement et réinsertion sociale), les structures associatives, les entreprises, les administrations, les organismes 
du Ministère de la Défense, les lieux de prise en charge judiciaire et de privation de liberté.

Les lieux d'intervention sont larges et variés ce qui permet de toucher un public plus nombreux notamment dans les Zones d'Education Prioritaires et les territoires ruraux.

Les objectifs et enjeux du service sanitaire sont donc multiples.

\section{Un objectif pédagogique}

En effet, avant la réalisation de son action de prévention, l'étudiant doit acquérir les connaissances nécessaires: en matière de santé publique (politique, organisation, promotion), de communication en fonction du public ciblé, pour pouvoir identifier les lieux susceptibles d'accueillir l'action de santé, afin d'utiliser au mieux les méthodes qualitatives et quantitatives d'analyse, les sources de données et d'informations, afin d'évaluer l'impact des actions menées au cours du stage.

Ces actions seront purement citoyennes et solidaires car la rémunération n'est pas envisagée pour ce nouveau module. Cependant, l'État prendra en charge les frais de déplacements. L'étudiant ne sera donc pas rémunéré pour ses interventions mais ces dernières ne devront rien lui coûter et être aménagées sur son temps de formation sans augmenter sa charge de travail.

A la fin du service sanitaire et de la réalisation de l'action de prévention, un temps de débriefing et d'évaluation permet d'échanger avec les référents du programme. L'auto-évaluation est donc primordiale.

Comme le souligne Eric Wolf, Professeur Principal de Collège, l'avantage de ce type d'action «c'est que ce sont des étudiants donc des jeunes qui parlent à d'autres jeunes. Le fait que ce soit des étudiants qui parlent, qui utilisent les mêmes mots permet aux élèves d'être moins complexés quand il faut parler de certaines pratiques, comme l'homosexualité, etc. Ce sont des sujets compliqués à aborder avec des adultes».

Le Service Sanitaire doit donc rendre la prévention accessible à tous par des moyens pédagogiques différents.

En choisissant, la prévention par l'intermédiaire du Service Sanitaire, le gouvernement souhaite également lutter contre les coûts générés par la morbidité évitable.

Le coût social des drogues licites ou illicites s'élevait en France à près de 250 milliards d'euros par an, estime l'économiste Pierre Kopp dans une étude publiée en 2015 effectuée pour l'Observatoire Français des Drogues et des Toxicomanies (OFDT). Les dépenses nettes de l'Etat liées aux drogues s'élevaient à 22 milliards d'euros, dont près de cinq milliards pour l'alcool, près de 15 milliards pour le tabac et plus 2,3 milliards pour les drogues illicites, soit 5,8\% des dépenses publiques, près d'un tiers du déficit budgétaire.

C’est aussi plus de 800.000 années de vie qui ont été perdues en raison des pathologies liées à la consommation d'alcool, plus de 680.000 à cause du tabagisme et plus de 40.000 à cause de l'usage de drogues illicites.

Le travail sur l'information et la prévention est donc primordial. L'enjeu du service sanitaire, nonobstant sa fonction première de prévention, est finalement une transformation à long terme de l'action des professionnels de santé par une modernisation du cursus des études et l'adaptation aux besoins de prévention de la France.

Il s'agit: d'initier tous les futurs professionnels de santé aux enjeux de la prévention primaire et de la promotion de la santé, de développer leurs compétences à mener des actions auprès de tous les publics, de lutter contre les inégalités territoriales et sociales en santé par des interventions auprès des publics les plus fragiles, de lutter contre la morbidité évitable, de favoriser l'autonomie des étudiants dans le cadre d'une pédagogie par projet et renforcer le sens de leur engagement dans leurs études, de favoriser l'inter-professionnalité et l'interdisciplinarité des étudiants en santé par la réalisation de projets communs à plusieurs filières de formation.

La mise en place d'une politique publique de service sanitaire à l'échelle nationale est inédite en Europe. Et comme le souligne le Pr Vaillant dans son rapport remis au gouvernement, «l'instauration d'un service sanitaire pour tous les étudiants en santé constitue une réforme ambitieuse qui concourt au virage préventif préconisé par le Haut Conseil de la Santé Publique et constitue un axe prioritaire de la stratégie nationale de santé 2018-2022. La réussite du service sanitaire qui amorce un changement culturel mettant la prévention au centre des pratiques des futurs professionnels de santé suppose un réel accompagnement du changement. Les acteurs nationaux et les acteurs de terrain ont ici un rôle majeur à jouer.

Les universités, écoles et instituts de formation des étudiants en santé, les Agences Régionales de Santé, les rectorats, les professionnels de la prévention, les associations qui œuvrent dans ces domaines auront à s'impliquer pleinement pour permettre au service sanitaire de s'exprimer dans toutes ses dimensions.

La dimension de réduction des inégalités territoriales et sociales en santé avec notamment des 
actions réalisées pour des publics en situation de précarité apparaît essentielle mais l'implémentation de ce type d'actions nécessitera probablement un peu plus de temps».

A long terme, le service sanitaire devra permettre de garantir la compétence de tous les professionnels de santé à mener des actions de prévention et de promotion de la santé auprès de tous les publics et de lutter contre les inégalités territoriales d'accès aux soins en développant des collaborations avec des professionnels variés qu'il soient liés au secteur de la santé ou non.

\section{La Formation des Infirmiers en France, un Référentiel Basé sur la Compétence}

La modernisation du système de santé français dans son ensemble impacte toutes les professions de santé et entraîne une nécessaire évolution des métiers de la santé et donc de la formation de ces futurs professionnels.

Le contexte actuel de la prise en soins à l'hôpital s'effectue au travers d'un ensemble coordonné d'acteurs de soins et de dispositifs médicaux, techniques, de procédures standardisées, de protocoles de soins. L'objectif est de rendre efficiente la prise en charge des patients, au moyen de l'utilisation des connaissances sans cesse en évolution, ce qui induit une réorganisation des structures de soins, des pratiques soignantes et de l'apprentissage.

Ainsi la formation des professionnels de santé s'ouvre vers le développement et l'actualisation de connaissances, de compétences spécifiques, en formation initiale et continue. Dans ce contexte de grande évolution des techniques médicales et scientifiques, la formation des futurs professionnels de santé nécessite l'ouverture et la réorganisation des référentiels de formation. Ainsi le passage de la qualification à la compétence a imposé la refonte des programmes de formation des professions para médicales.

En 2009, le ministère de la santé a entrepris la rénovation du diplôme infirmier, selon un modèle permettant son inscription dans la structure universitaire du processus de Bologne et lui conférant une certification européenne par l'obtention d'un grade Licence.

La réforme de la formation des Infirmiers Diplômés d'État de 2009 (DEI) (arrêté du 31 juillet 2009 relatif au DEI), permet l'entrée de cette profession dans le cadre du système Licence-Master-Doctorat (LMD) et l'obtention du grade de licence au diplôme d'Etat d'Infirmier, grade qui permet désormais de poursuivre un parcours en master et en doctorat.

Pour acquérir les compétences indispensables à l'exercice du métier, trois années de formation découpées en six semestres (équivalant à 4200 heures) sont nécessaires à l'étudiant en soins infirmiers. La sélection des candidats à la formation des infirmiers s'opère après l'obtention du baccalauréat.

Le dispositif de formation repose sur un référentiel de compétence issu du référentiel d'activités basé sur la fiche métier du répertoire des métiers de la fonction publique.

Cinq compétences constituent le «cour de métier»:

- Évaluer une situation clinique et établir un diagnostic dans le domaine des soins infirmiers;

- Concevoir et conduire un projet de soins infirmiers ;

- $\quad$ Accompagner une personne dans la réalisation de ses soins quotidiens ;

- Mettre en œuvre des actions à visée diagnostique et thérapeutique ;

- Initier et mettre en œuvre des soins éducatifs et préventifs.

Cinq compétences "transverses», communes à certaines professions paramédicales:

- Communiquer et conduire une relation dans un contexte de soins;

- Analyser la qualité des soins et améliorer sa pratique professionnelle;

- Rechercher et traiter des données professionnelles et scientifiques;

- Organiser et coordonner des interventions soignantes;

- Informer et former des professionnels et des personnes en formation.

En France, la formation des infirmiers est une formation en alternance constituée à part égale d'une formation clinique en milieu professionnel et d'une formation théorique.

- La formation théorique de 2100 heures, sous la forme de cours magistraux, de travail personnel guidé et de travaux dirigés et de pratique des soins par la simulation.

- La formation clinique de 2100 heures, en milieux professionnels en lien avec la santé et les soins. «Ces périodes alternent avec les périodes d'enseignements théoriques en instituts de formation. Pendant ces temps de stage l'étudiant se trouve confronté à la pratique soignante auprès des patients... ${ }^{7}$.

Dans ce référentiel de formation la notion de compétence est essentielle et nécessite de bien la cerner. Ainsi dans ce nouveau dispositif, développer des compétences ce n'est pas accumuler des connaissances, des habiletés gestuelles, organisationnelles, techniques, des capacités. Mais c'est 
être capable de combiner tout cela en situation et de pourvoir aussi le transférer.

\section{Alors de quoi parlons-nous lorsque nous parlons de compétence?}

«La compétence d'un professionnel se reconnaît à sa capacité à gérer efficacement un ensemble de situations professionnelles. Pour cela il devra combiner et mobiliser plusieurs compétences ou ressources ${ }^{8}$. La compétence se reconnaît et serait le résultat d'une mobilisation de ressources transférables dans différentes situations réelles.

C'est la mobilisation de plusieurs ressources, de différents savoirs, des «savoirs théoriques (savoir comprendre, savoir interpréter), de savoirs procéduraux (savoir comment procéder), savoir-faire procéduraux (savoir procéder, savoir opérer), savoir-faire expérientiels (savoir y faire), savoir-faire sociaux (savoir se comporter, se conduire), de savoir-faire cognitif (savoir traiter de l'information, savoir raisonner, savoir nommer ce que l'on fait, savoir apprendre)» ${ }^{9}$. Il s'agit alors de pouvoir aménager de façon adéquate et efficace ces ressources en situation.

Ainsi nous pouvons considérer que la compétence s'acquiert au fil des savoirs acquis et mobilisés lors d'expériences précédentes, de situations de soins vécues auprès du patient. Ces savoirs, transférables, permettent alors la gestion de situations nouvelles pour lesquelles nous n'avons pas de repère établi, de référence. Ainsi nous pouvons dire que la compétence se construit.

Et en même temps, le premier sens du mot compétence est issu du vocabulaire juridique, en tant qu'aptitude reconnue légalement à une autorité publique d'agir dans des conditions déterminées. De là découle l'idée d'aptitude, d'habileté reconnue à quelqu'un du fait de ses connaissances et de son expérience, cette reconnaissance lui conférant le droit de juger ou de décider.

Dans ce cadre, la compétence est l'aptitude que possède une personne pour porter un jugement de valeur dans un domaine dont elle a une connaissance approfondie, c'est ce que l'on appelle la compétence professionnelle ${ }^{10}$. Pour que la compétence puisse exister il faut que le pouvoir d'agir et de décider de façon appropriée soit reconnu par autrui.

D’un autre côté, «la compétence ne se caractérise pas par l'atteinte d'un objectif fixé d'avance, en obtenant les meilleurs résultats possibles; ce qui la caractérise, c'est la pertinence, c'est-à-dire le fait de convenir exactement à l'objet dont il

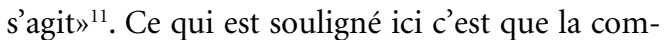
pétence de l'infirmier, du soignant se caractérise par la pertinence du soin qu'il prodigue à un patient lors d'un colloque singulier et non pas la validité théorique, virtuelle, d'un soin standardisé, non singulier qui ne permet pas de mettre en scène le colloque singulier.

\section{Ainsi, lorsque l'on parle d'amélioration des compétences, de quoi parlons-nous?}

Les soins infirmiers sont très diversifiés et comportent de nombreux aspects techniques, organisationnels, scientifiques et dans tous les cas relationnels qui requièrent des compétences spécifiques.

Nous pouvons distinguer trois types de compétences, «les compétences conceptuelles (analyser, comprendre, agir de manière systémique), les compétences techniques (méthodes, processus, procédures, techniques d'une spécialité), les compétences humaines (dans les relations intra et interpersonnelles)» ${ }^{12}$.Ce type de découpage, plus classique, est un repère qui semble bien adapté au domaine des soins infirmiers et décompose les compétences en savoirs, savoir-faire et savoir être.

L'exploration des différentes manières d'aborder la compétence montre deux conceptions opposées, l'une behavioriste, centrée sur les savoirs, l'autre centrée sur l'apprenant et le développement de ses potentialités internes.

La conception béhavioriste souligne l'apprentissage de "conduite de comportements structurés en fonction d'un but, d'une action, d'une tâche spécifique, observable et qui repose plus sur des savoirs et contenus de programme» ${ }^{13}$. Cette conception semble adaptée à l'apprentissage de procédures, de protocoles. Classiquement la compétence est envisagée comme le fruit de l'apprentissage long, fruit de l'expérience, de l'ancienneté reconnue aux personnes. Pourtant cette conception donne ici le primat à l'image du «moule» qui imprime sa forme sur l'individu presque malgré lui, l'idée que la compétence s'est incorporée à l'homme comme une seconde nature.

D'un autre côté, la conception classique de la compétence se déconstruit face à la conception qui aborde la compétence comme potentiel à développer reconnu par des résultats et qui donne ici une prééminence au savoir à développer; sa reconnaissance étant conditionnée par des résultats.

\section{Mais qu'en est-il de l'apprentissage de l'adaptation de ces procédures, de ces protocoles à la singularité du patient?}

Dans le domaine du soin la compétence permet de prendre en soins le patient dans sa singu- 
larité et dans la spécificité des circonstances, des événements. Elle permet d'agir de façon adaptée au bon moment de saisir le kairos.

Lorsque nous envisageons la compétence à la manière «d'une potentialité intérieure, invisible, une capacité générative susceptible d'engendrer une infinité de conduites adéquates à une infinité de situations nouvelles» ${ }^{13}$; nous envisageons la compétence davantage comme traduction du fonctionnement cognitif de l'apprenant, du sujet, que comme manifestation d'un savoir.

Aussi lors de la fabrication de la compétence $^{14}$, il se produit un changement du rapport aux choses qui affecte autant les savoirs mobilisés que l'individu qui les développe. Cette transformation est envisagée comme impression sur la matière, à la manière du moule et aussi comme développement des potentialités.

En opposition au temps long de l'expérience, la conception du temps court de la compétence est conçue «comme mobilisation de diverses capacités de manière intégrée dans le sillage du triptyque du savoir, savoir-faire, savoir être» ${ }^{15}$. Ainsi sorti de la conception béhavioriste, et la métaphore du moule, nous allons vers la compétence en tant que processus permettant d'aboutir à un résultat inédit, singulier.

Et c'est bien de ce type de compétence dont l'infirmier a besoin pour s'adapter à la singularité du patient, du contexte et aux évolutions technoscientifiques.

Ainsi, la compétence en tant que processus s'établit à partir de la mobilisation de différentes ressources, des potentialités initiales, qui par «capillarité» conduisent à des transformations pour aboutir à un résultat singulier.

Les pédagogies actives, telle la formation en simulation, l'analyse de pratiques, permettent le développement de ce processus, car l'enjeu est moins la transmission de savoir que la construction de savoir par l'apprenant lui-même.

La question, maintenant, porte sur la nature des ressources à mobiliser pour que le résultat soit adapté et de savoir si ces ressources sont mobilisables lors de l'apprentissage en simulation.

La prise en soins repose sur les mobilisations de multiples savoirs qui ne sont pas que d'ordre cognitif mais également d'ordre procédural et émotionnel. Bien que les conditions de l'aménagement adéquat de ses ressources en situation peuvent rester assez mystérieuses, nous comprenons aisément que si être compétent c'est être capable d'agir, de décider efficacement et être reconnu pour cela, l'utilisation de techniques pédagogique comme l'apprentissage en simulation est bien adaptée au développement nécessaire, de l'intelligence logique et rationnelle, importante pour assurer un travail méthodique, planifié, ordonné et sécuritaire. Par ailleurs, l'observation du professionnel infirmier en situation professionnelle couplée aux savoirs rationnels, par raisonnement inductif, nous permet aussi d'aller chercher des données relatives au vécu du malade. La sélection et la mise en lien de ces données nous permettent alors de résoudre des problèmes et de planifier des soins adaptés et bien ciblés.

Nos rapports humains avec les malades sont complexes et exigent pour saisir leur réalité, un ensemble de raisonnements et de perceptions et donc d'émotions, les émotions vécues lors de la rencontre, cette intelligence émotionnelle assure aussi l'efficacité de nos actions, de nos relations.

L'apprentissage de la pratique est un des socles importants de la formation infirmière. L'évolution des besoins en santé des populations, le bouleversement technoscientifique des prises en charges médicales confrontent le soignant à des situations de soins complexes, à cela s'ajoute l'augmentation de l'exigence de qualité et d'efficience.

La finalité du programme de formation des infirmiers est de former un futur professionnel autonome et réflexif qui doit être capable d'autoévaluer ses pratiques, d'apprendre en situation et par la situation.

Devant un domaine en perpétuel évolution comme celui de la santé il semble nécessaire que le professionnel de santé sache remettre en question autoévaluer ses pratiques et ses savoirs. C'est l'analyse de pratiques qui permet ce travail, elle se réalise en situation réelle auprès du patient lors des stages, mais aussi en situations simulées, en laboratoire de simulation. L'idée étant de favoriser un apprentissage par l'activité pour que les étudiants puissent construire, intégrer les nouveaux savoirs et les transférer.

Dans les instituts de formations en soins infirmiers l'apprentissage par la simulation, promu par la H.A.S ${ }^{16}$ comme méthode prioritaire pour la formation initiale et continue des professionnels de santé, peut concerner de nombreuses activités intégrant une mise en situation clinique et fait appel à des simulateurs techniques, plus ou moins élaborés, pour la répétition de gestes techniques et aux jeux de rôle.

Pourtant, si nous voulons permettre à l'étudiant de développer des compétences nous ne pouvons pas nous arrêter à la seule répétition de gestes conformes au protocole, un schéma béhavioriste de reproduction, notamment lorsque nous évaluons en situation simulée la préparation d'une injection, la réalisation d'une transfusion, l'injection sur chambre implantable ${ }^{7}$. 
Pour aller vers la compétence il est nécessaire de sortir de cette idée métaphorique du moule pour aller vers une compétence en tant qu'adaptation au singulier du contexte, de l'environnement, du patient au travers d'un processus de transduction des savoirs qui de proche en proche permet d'aboutir à un résultat singulier adapté à chaque patient.

Finalement ce qui importe pour développer des compétences et se professionnaliser c'est l'analyse réflexive, la situation simulée n'étant que le support, le prétexte à celle-ci. L'analyse réflexive des différentes activités réalisées permet le développement de compétences et la structuration de son identité professionnelle.

Lors des apprentissages en milieu clinique, l'infirmier en exercice montre, fait avec l'étudiant stagiaire, laisse faire sous son regard, explique les raisons de ses choix, de ses stratégies; ce que nous appelons la face invisible du soin, questionne l'étudiant pour savoir ce qu'il a compris, réalise avec lui un retour réflexif.

Poser des questions à la manière d'Aristote et de sa maïeutique, faire «accoucher» les esprits, permet de faire exprimer le savoir caché, ce que l'on ne sait pas que l'on sait. Ainsi la qualité de l'apprentissage en milieu de soin permet à l'étudiant de dépasser le mimétisme, reproduire ce qu'il voit, de mettre en évidence le processus invisible qu'est le raisonnement clinique qui mène au soin.

Par ailleurs, l'épanouissement de l'intelligence émotionnelle est primordiale pour la relation soignante car elle humanise le soin ${ }^{17}$. Cette forme d'intelligence est complémentaire au raisonnement logique et doit aussi être travaillée dès la formation initiale. Au-delà des qualités de l'intelligence rationnelle, indispensables pour assurer une prise en soin organisée et sécuritaire, l'apport des émotions lors des interactions donnent du sens à la relation et humanisent le soin. Les relations avec les patients sont complexes et exigent pour saisir leur réalité la mise en jeu d'un ensemble de perceptions, d'émotions qui vont nous guider dans nos interactions. Cet ensemble fait appel à d'autres formes d'intelligences, l'intelligence émotionnelle et l'intelligence sociale qui nous permettent de comprendre notre environnement, de nous adapter, de réaliser ce que ce que vit l'autre.

\section{Apprendre à prendre soin}

Pour prendre soin au quotidien, il n'est pas nécessaire d'avoir un diplôme ${ }^{18}$, les parents prennent soin de leurs enfants, nous prenons soin des personnes qui nous sont chers et même des objets lorsqu'ils ont une valeur émotionnelle. Prendre soin de quelque chose, de quelqu'un c'est se préoccuper de, c'est l'expression d'une attention particulière, de l'importance que nous accordons à l'autre.

Former des futurs professionnels de santé c'est aussi les sensibiliser, développer en eux cette disposition au prendre soin. Tandis que prendre soin ne suffit pas seulement pour être soignant, il faut encore savoir faire des soins et pour faire des soins il faut nécessairement avoir un diplôme, être formé.

Pour cela le formateur, l'enseignant doit faire preuve de créativité, d'ingéniosité pédagogique pour associer les techniques de soins, au prendre soin.

Former des professionnels de santé, c'est prendre le soin d'éduquer aux soins, la mise en relation d'un savoir au service d'autrui et d'une pratique, qui n'est pas seulement l'application d'un savoir.

Bien que la simulation en tant que technique pédagogique est un outil utile lorsqu'elle permet de soumettre l'apprenant à des situations réalistes avant de pratiquer sur les patients et qu'elle permet le développement de l'esprit critique, elle se borne trop souvent à des objectifs sécuritaires, l'apprentissage de protocoles techniques sur le mode de l'évidence based teaching en référence à evidence based nursing.

Prendre soin ne nécessite pas seulement d'être un bon technicien, il faut aussi de l'intelligence émotionnelle. Les infirmiers sont des hommes et des femmes portés par le sentiment d'appartenance à une culture soignante où le savoir-faire humain attentif à l'autre est inspiré par le regard porté à l'autre, au corps, à l'environnement dans un respect mutuel entre êtres humains.

Au même titre que soigner, éduquer est un art, un savoir-faire touchant, au-delà des apprentissages, à l'action de l'homme sur l'homme. Cet aspect de la tèchnè, l'art de savoir dans l'éducation et dans les soins nous laisse entrevoir que former et éduquer sont des métiers du care, une forme d'attention à l'autre qui marque notre dépendance et notre vulnérabilité.

Platon se réfère souvent à la médecine comme modèle pour la politique et pour Aristote la médecine est une mine de pratiques et de savoirs. Pour lui l'art de soigner est l'illustration du « juste milieu », la médecine, comme l'éducation n'étant pas seulement connaissance, savoir mais aussi pratique d'une activité singulière, dont la finalité est de former, de soulager, de guérir et non le savoir en lui-même. Une tèchnè qui se construit 
par l'expérience et génère la poïétique adaptée au cas singulier, un art en somme.

Ainsi, nous donnons aux acteurs qui forment les professionnels de santé, le soin d'éduquer aux soins, deux termes qui reposent sur la mise en relation d'un manque avec une compétence au service d'autrui, la mise en relation d'un savoir et d'une pratique où la pratique n'est pas seulement l'application d'un savoir.

Former, et se former c'est donner du sens et ressentir des émotions. Donner du sens, un terme qui peut paraître galvaudé, pavé de bonnes intentions, nous dirons alors, que former aux soins c'est essayer de faire saisir l'essence du soin, donner de la consistance à l'action de soigner et ressentir des émotions.

\section{La Démographie des Infirmiers en France}

Le nombre d'infirmiers en France s'élèverait à 638.248 (contre 616.573 en 2014), selon le rapport «La démographie des autres professions de santé (RPPS et Adeli)» publié par la Direction de la recherche, des études, de l'évaluation et des statistiques (Drees) le 8 avril 2015. Ainsi, ils seraient 109.925 à exercer en libéral, et 528.323 à être salariés.

La profession dénombre également $87 \%$ de femmes ( $84 \%$ dans le milieu libéral et $88 \%$ de salariées). Les infirmiers spécialisés représentent quant à eux 52.463 professionnels, dont:

- 19.074 infirmiers(ères) puériculteurs (trices);

- 9.709 infirmiers anesthésistes;

- 7.225 infirmiers de bloc opératoire;

- $\quad 9.307$ cadres de santé;

- 423 infirmiers cadre de santé publique;

- 5.995 cadres infirmiers;

- $\quad 730$ cadres infirmiers psychiatriques.

Près de la moitié des infirmiers (322.996) exercent au sein d'hôpitaux publics. Viennent ensuite les établissements de santé privé lucratif (69.438) puis les cabinets individuels (61.768).

Les zones d'activités comptant le plus d'infirmiers sont les zones aux abords des grandes villes francaises Paris, Lyon, Marseille. Les régions dénombrant le moins d'infirmiers sont, hormis les DOM-COM, la Corse (3.368), le Limousin (9.733), et la Franche-Comté (11.909).

Les statistiques concernant la démographie des professions de santé publiées par la DREES portent sur les professionnels inscrits dans le répertoire Adeli ou dans le Répertoire Partagé des Professionnels de Santé (RPPS) comme étant actifs occupés au 1er janvier.

Pour rappel, la Direction de la Recherche, des Études, de l'Évaluation et des Statistiques (DREES) recense, pour l'année 2017, 10.311 IADE dont $69 \%$ de femmes et $31 \%$ hommes. Selon les résultats de l'enquête du SNIA, 40-44

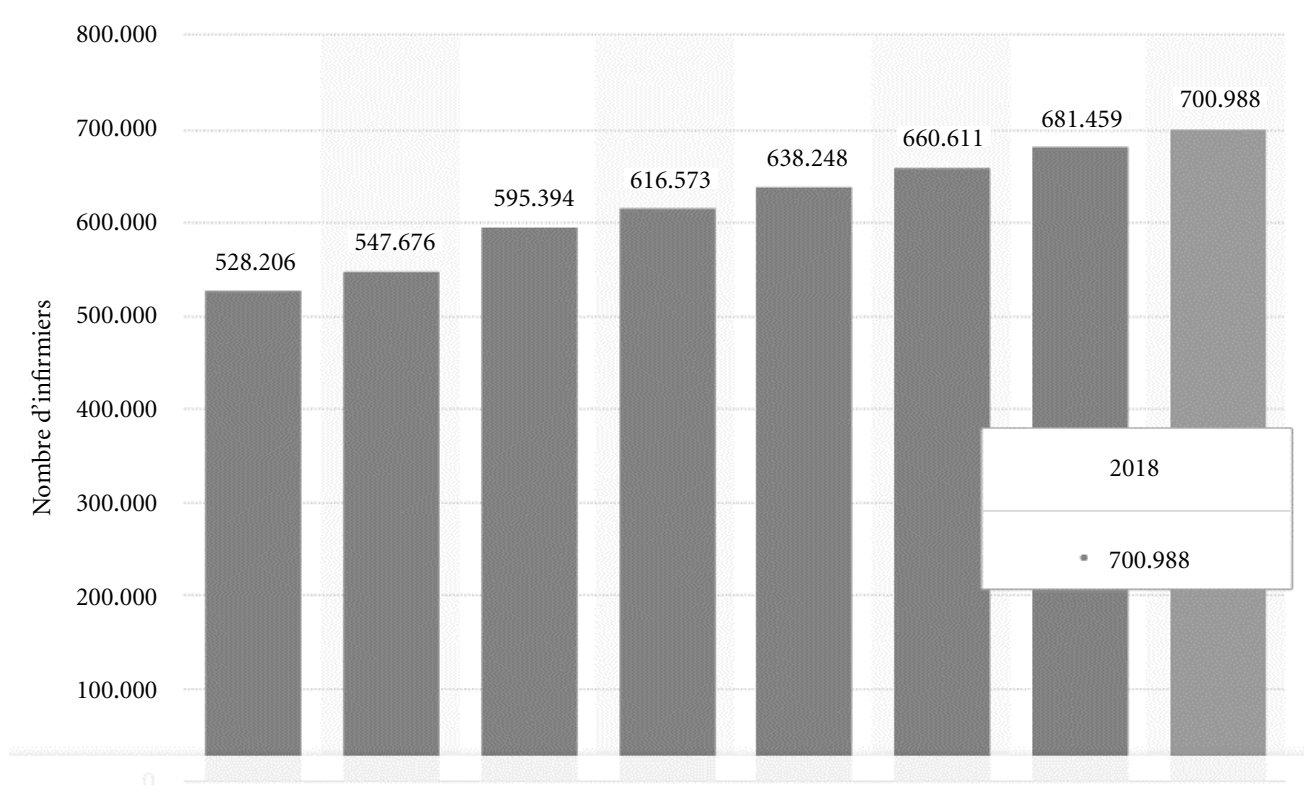

Graphique 1. Nombre d'infirmiers exerçant en France de 2010 à 2018. 
ans est la fourchette en âge la plus représentée (23,25\%), suivie de $35-39$ ans $(18,09 \%)$ et de 49 47 ans $(17,97 \%)$. On note également une surreprésentation significative des hommes de 40-44 ans ( +13 points) et une sous-représentation des professionnels de plus de 60 ans.

La répartition du panel selon les fonctions exercées se compose comme suit : IADE (93,06\%), Cadres de Santé (5,63\%), IADE Cadres Supérieurs de Santé (1,20\%), IADE Directeurs des Soins soit $(0.11 \%)$.

Cette statistique ${ }^{19}$ indique le nombre d'infirmiers exerçant en France entre 2010 et 2018
(Graphique 1). On remarque que le nombre d'infirmiers et infirmières a augmenté durant cette période, dépassant 600.000 infirmiers en 2014.

\section{Collaborateurs}

C Guesdon-Caltero: conception, design et rédiger. N Cherchem: conception, design et rédiger. MA Frota: rédiger et commenter de manière critique. KMC Rolim: Approbation de la version à publier.

\section{Références}

1. Anjos JN. Regulação no Setor da Saúde: Análise de Direito Comparado - Portugal, Reino Unido, França Espanha [Internnet]. Coimbra: Publicações CEDIPRE Online; 2015. [acessado 2015 Dez 26]. Disponível em: https://www.fd.uc.pt/cedipre/wp-content/uploads/ pdfs/co/public_26.pdf

2. Cordilha AC, Lavinas L. Transformações dos sistemas de saúde na era da financeirização. Lições da França e do Brasil. Cien Saude Colet 2018; 23(7):2147-2158.

3. Teixeira VMN, Cunha YMM. A pesquisa em História da Enfermagem: revisão de publicações de 2000-2008. REME 2011; 15(3):435-442.

4. Padilha MICS, Borenstein MS. História da Enfermagem: ensino, pesquisa e interdisciplinaridade. Esc Anna Nery 2006; 10(3):532-538.

5. Courtois AC, Courtois R, Cuminet L, Grandsire A. Quelle est l'image de l'infirmière aujourd' hui? Soins 2005; 700: 41-47.

6. Isambart G. Interview exclusive de la première présidente de l'ordre des infirmiers. Infirmiers.com; 2009. [acessado 2019 Jul 16]. Disponível em: https://www. infirmiers.com/les-grands-dossiers/ordre-infirmier/ interview-exclusive-de-la-premiere-presidente-delordre-des-infirmiers-dominique-le-boeuf.html

7. Collectif. Profession Infirmier. Recueil des principaux textes relatifs à la formation préparant au diplôme d'état et à l'exercice de la formation de la profession. Paris: Berger-Levrault; 2015.

8. Le Boterf G. De quel concept de compétence avonsnous besoin? Soins Cadres 2002; 41:20-22.

9. Le Boterf G. De la compétence, essai sur un attracteur étrange. Paris: Editions d'organisations; 1995.

10. Centre National de Ressources Textuelles et Lexicales (CNRTL) [página na Internet]. [acessado $2019 \mathrm{Jul}$ 16]. Disponível em: https://www.cnrtl.fr/etymologie/

11. Péaud P. Espace pédagogique de l'académie de Poitiers. Un peu d'analyse sémantique et étymologique [página na Internet]. 2011 [acessado 2019 Jul 16]. Disponível em: http://ww2.ac-poitiers.fr/competen ces/spip.php?article126
12. Katz RL. Skills of an effective administrator. Harvard Business Review 1974; 52(5): 90-102.

13. Frenay $M$, Noël B, Parmentier P, Romainville $M$ L'étudiant-apprenant. Grilles de lecture pour l'enseignant universitaire. Revue française de pédagogie 1999; 127(1):170-171.

14. Stroobants M. Savoir-faire et compétence au travail. Une sociologie de la fabrication des aptitudes. Bruxelles: Editions de l'Université libre de Bruxelles; 1993.

15. Monchatre S. Déconstruire la compétence pour comprendre la production des qualifications [artigo na Internet]. Revue ¿Interrogations?; 2010. [acessado 2017 Maio 27]. (La compétence no 10). Disponível em: http://www.revue-interrogations.org/Deconstruire -la-competence-pour

16. Haute Autorité de Santé (HAS) [página na Internet]. [acessado 2017 Maio 27]. Disponível em: https:// www.has-sante.fr/

17. Phaneuf M. l'intelligence émotion: dossier le vécu émotionnel. Santé Mentale 2013; 177:30-35.

18. Hesbeen W. Conférence, Master de Philosophie. Parcours éthique médicale et hospitalière appliquée. Promotion 2015-2017. Année 2016-2017.

19. Statista Research Department. Dernière mise à jour le 1 mars 2019 [página na Internet]. [acessado 2019 Jul 16]. Disponível em: https://fr.statista.com/statistiques/616593/effectifs-infirmiers-france/

Artigo apresentado em 30/04/2019

Aprovado em 20/08/2019

Versão final apresentada em 25/09/2019 